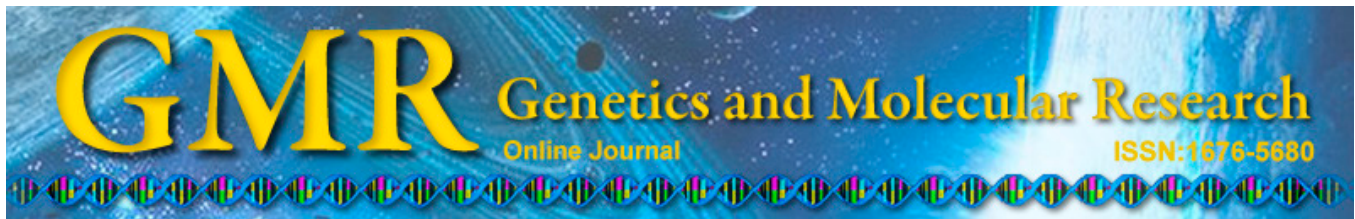

\title{
Correlation between single nucleotide polymorphisms in hypoxia-related genes and susceptibility to acute high-altitude pulmonary edema
}

\author{
A.L. Wu, Y.S. Xiong, Z.Q. Li, Y.G. Liu, Q. Quan and L.J. Wu \\ Research and Service Center of Laboratory Medicine, \\ Chengdu Military General Hospital, Chengdu, Sichuan, China \\ Corresponding author: L.J. Wu \\ E-mail: 379197283@qq.com
}

Genet. Mol. Res. 14 (3): 11562-11572 (2015)

Received January 28, 2015

Accepted May 26, 2015

Published September 28, 2015

DOI http://dx.doi.org/10.4238/2015.September.28.8

ABSTRACT. This study aimed to explore the relationship between
genetic changes and high-altitude pulmonary edema (HAPE)
susceptibility, and to screen for the key single nucleotide polymorphism
(SNP) loci in the HAPE-susceptibility gene, by investigating the
SNPs occurring in hypoxia-related genes in HAPE-susceptible and
control (non-susceptible) populations. This research was conducted
on Han recruits, who travelled to the Lhasa plateau (altitude, 3658
m). Ten loci located on ten genes extracted from the HAPE and
healthy populations were amplified by polymerase chain reaction, and
subsequently sequenced. The investigated genes included those coding
for aldosterone synthase 2 (CYP11B2), angiotensin-converting enzyme
(ACE), heat-shock protein 70 (HSP70), nuclear factor kappa B (NF-
אB), surfactant protein A2 (SP-A2), plasminogen activator inhibitor-1
(PAI-1), nitric oxide synthetase (NOS), vascular endothelial growth
factor (VEGF), prolyl hydroxylase (EGLN1), and zinc finger protein
A20. The gene distribution of each SNP loci and its correlation with 
HAPE was analyzed. Statistical analyses of the genotype frequencies of the SNPs revealed significant differences in the $A C E$ (rs4309), EGLN1 (rs480902), SP-A2 (rs1965708), HSP70 (rs1008438), PAI-1 (rs 1799889), and NOS (rs199983) expressions between the HAPE and healthy control groups $(\mathrm{P}<0.05)$; therefore, these SNP loci were believed to indicate HAPE susceptibility. HAPE is correlated with multipleSNP loci. A correlation analysis between genetic polymorphism and HAPE susceptibility revealed that 6 hypoxia-related genes were key sites accounting for HAPE. These findings could help assess the risk of HAPE in populations expressing different genotypes, in order to reduce the occurrence of HAPE.

Key words: HAPE; Heat-shock protein 70; Prolyl hydroxylase; Angiotensin-converting enzyme; Surfactant protein A2;

Plasminogen activator inhibitor

\section{INTRODUCTION}

High-altitude pulmonary edema (HAPE) is a type of pneumonedema that specifically occurs under conditions of high altitude and hypoxia. This disease commonly occurs in plainsmen who move to/enter a plateau, or in plateau residents who travel to higher altitude areas; the incidence of HAPE in such populations has been determined to be $0.4-2 \%$ (Gao and Gao, 2009). HAPE is an acute onset disease that is known to proceed rapidly; therefore, timely diagnosis and treatment of HAPE is required to counteract its harmful effects, including the incidence of coma or even death (within a short period of time) (Gao, 2005). Recent studies investigating HAPE have focused on multiple in vivo systems, such as the cell ion transport and endothelial systems (Saxena et al., 2005; Manuguerra et al., 2006; Meyer et al., 2007; Qi et al., 2008). Despite this, the etiology of HAPE remains unclear; in addition, there is a lack of reliable methods for predicting the occurrence of HAPE in susceptible individuals (who travel to plateaus). The occurrence of HAPE is affected by many factors, including environmental and genetic factors. However, susceptibility to the environment is governed by genetic factors (Patel and Peacock, 2001; Hotta et al., 2004). This study investigated and compared the characteristics of clinical HAPE patients and healthy individuals; the relationship between the single nucleotide polymorphisms (SNP) of 10 candidate genes and HAPE was investigated to elucidate the genetic basis of HAPE formation and development. Through this, we attempted to identify the key gene regulating HAPE susceptibility, and provide an objective basis for the clinical prevention of HAPE.

\section{MATERIAL AND METHODS}

\section{Research subjects}

This study was conducted on 1200 new recruits from the Han ethnicity. All the subjects were male, aged between 18 and 20 years, and medically declared to be healthy prior to joining the army. The recruits were native to regions of different altitudes $(23,110$, 396 , and $1891 \mathrm{~m}$ ); they were made to travel to Chengdu, which was $505 \mathrm{~m}$ above sea level 
(Chengdu) by train. The subjects rested for 7 days in Chengdu, and subsequently traveled by plane to Lhasa (altitude: $3658 \mathrm{~m}$ ). Unless specified, this study classified a plain and plateau as regions that were 505 and $3658 \mathrm{~m}$ above sea level, respectively. One hundred and three patients, from among those meeting the HAPE Lake Loulse standard diagnostic score, were randomly classified into the HAPE group; two hundred recruits, from among those who did not meet the HAPE Lake Loulse standard diagnostic score, were selected as controls (Draft recommended by the Third National Congress of Chinese Medical Association for discussion on plateau medicine, 1996). All factors (service time, height, weight, body condition, ancestral home) were almost similar among the two groups, excluding the study factors. The study and all experimental protocols were approved by the Ethics Committee of the Chengdu Military General Hospital. Signed informed consent forms were obtained from all study subjects.

\section{SNP site selection}

Susceptible target genes and loci indicating acute discomfort as a result of exposure to plateau regions (mainly HAPE) were identified in the publicly available human genome data, obtained from the National Center for Biotechnology Information (NCBI) GenBank database, and through extensive literature research. Ten susceptibility genes and loci associated with HAPE were identified following extensive comparative bioinformatic analyses: $A C E$ (rs4309), CYP11B2 (rs1799998), EGLN1 (rs480902), SP-A2 (rs1965708), NF- $\kappa B$ (rs28720239), HSP70 (rs1008438), VEGF (rs3025039), PAI-1 (rs1799889), NOS (rs199983), and A20 (rs5029924). The target genes were selected based on the following principles: the gene frequency of each SNP loci was greater than 0.1 ; each SNP loci was located in the coding or regulatory regions, or region determining protein activity, of the gene; the genotype frequency information (obtained by literature reviews) was specific to the Chinese Han population.

\section{Major experimental materials}

The PrimeSTAR HS DNA polymerase PCR Kit, PrimeScript RT Kit, and the SYBR Premix EX Taq fluorescence quantitative PCR kit was obtained from TaKaRa Bio Inc. (Otsu, Japan). The gel extraction purification kit, column total RNA extraction kit, and all primers used in this study were obtained from Sangon Biotech (Shanghai, China).

\section{Genotyping}

\section{Genomic DNA extraction}

Peripheral venous blood was collected in the morning (early) from all subjects of both groups, into an ethylene diamine tetraacetic acid (EDTA)-K2 anticoagulant tube. Genomic DNA was extracted from these blood samples by the phenol chloroform method. The purity and concentration of the obtained DNA samples was determined.

\section{Amplification and purification of target gene}

The target gene was amplified on the ABI 2720 PCR machine (Applied Biosystems, Foster City, CA, USA). The primers were designed using the Primer 5 software, and their 
specificity confirmed by comparative analysis using the NCBI Basic Local Alignment Search Tool. All primers were synthesized by Sangon Biotech. The amplification was performed in a $50-\mu \mathrm{L}$ reaction mixture, containing $25 \mu \mathrm{L} 2 \mathrm{X}$ PrimeSTAR GC buffer, $0.5 \mu \mathrm{L}$ PrimeSTAR HS DNA polymerase, $4 \mu \mathrm{L}(2.5 \mathrm{mM}) \mathrm{dNTP}$ mixture, $1 \mu \mathrm{L}$ of each primer $(10 \mu \mathrm{M})$, and $\mathrm{H}_{2} \mathrm{O}$. PCR was performed under the following reaction conditions: denaturation at $95^{\circ} \mathrm{C}$ for $3 \mathrm{~min}$, 35 cycles of denaturation at $94^{\circ} \mathrm{C}$ for $30 \mathrm{~s}$, annealing at $59^{\circ} \mathrm{C}$ for $35 \mathrm{~s}$, and extension at $72^{\circ} \mathrm{C}$ for $50 \mathrm{~s}$, and a final extension at $72^{\circ} \mathrm{C}$ for $5 \mathrm{~min}$. The PCR products were electrophoresed on a $1.5 \%$ agarose gel, purified, and recovered using the column DNA gel extraction kit.

\section{DNA sequencing}

The PCR products were sequenced using the ABI 3730 analyzer (Applied Biosystems).

\section{Statistical analysis}

The obtained data were subjected to the $\chi^{2}$ test to analyze the Hardy-Weinberg equilibrium. The genotype distribution among patients was analyzed by $\chi^{2}$ and Fisher exact tests. P values $<0.05$ were considered to be statistically significant.

\section{RESULTS}

\section{Genotype distribution of each SNP locus in the hypoxia-related gene}

\section{Genotyping by sequencing}

Figures 1-10 show the sequencing peak diagrams of the SNP genotype; all sequencing peaks were observed to be clear, and with no obvious impure peak. The peaks were observed to be stable, and each genotype could be clearly distinguished. Each site could clearly distinguish the three genotypes, while the rs28720239 sites could clearly distinguish the two genotypes ATTG/-, ATTG/ATTG.

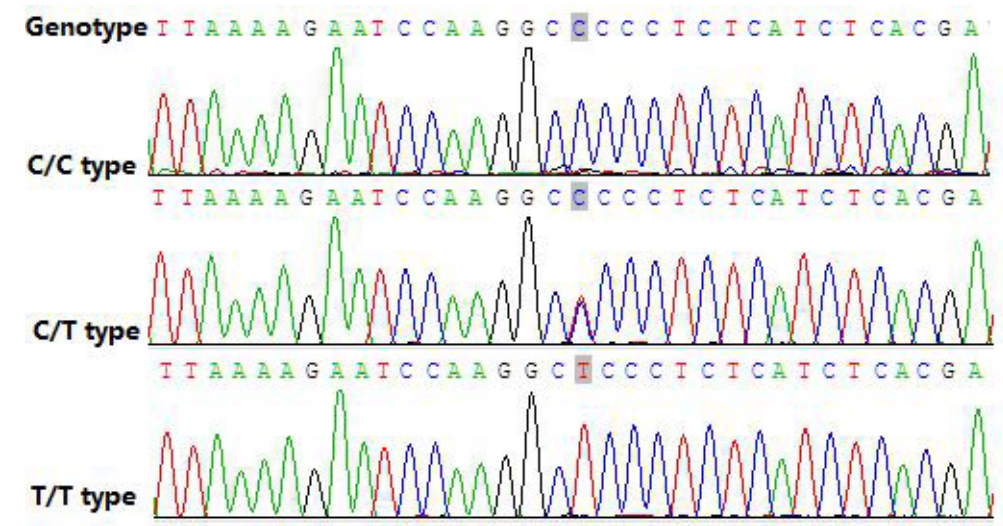

Figure 1. Aldosterone synthase 2 (CYP11B2; rs1799998). C/C type: wild homozygous, C/T: mutant heterozygous, T/T: mutant homozygous. 
Genotype CAGIACAAGG AI CI GC C C I C I C C I G CG I C G G
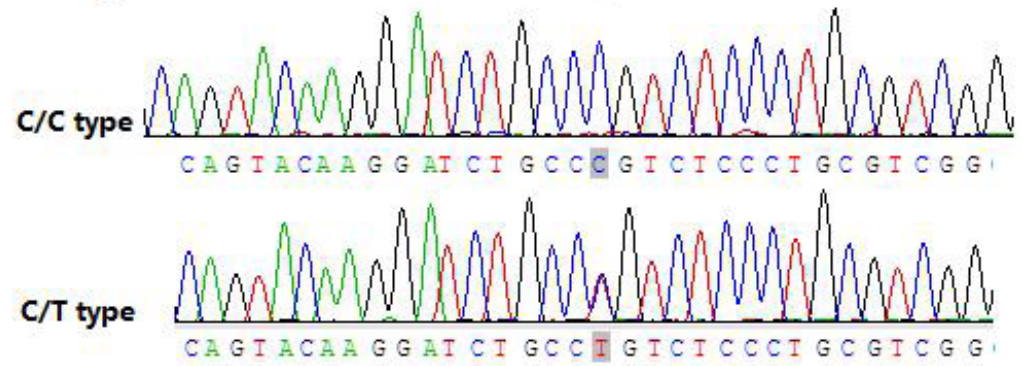

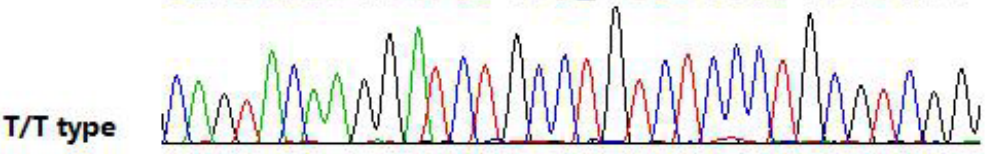

Figure 2. Angiotensin-converting enzyme ( $A C E$; rs4309). C/C type: wild homozygous, $\mathrm{C} / \mathrm{T}$ : mutant heterozygous, T/T: mutant homozygous.

$$
\text { Genotype CGGG A A A I I C CA GGG II I I C GCC I C C CG I }
$$
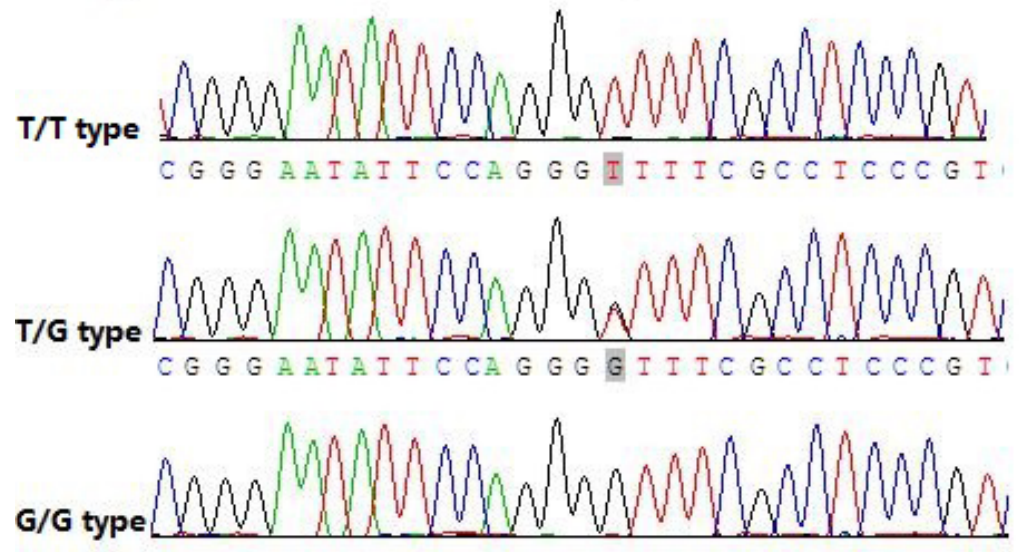

Figure 3. Heat shock protein 70 (HSP70; rs1008438). T/T type: wild homozygous, T/G: mutant heterozygous, G/G: mutant homozygous.

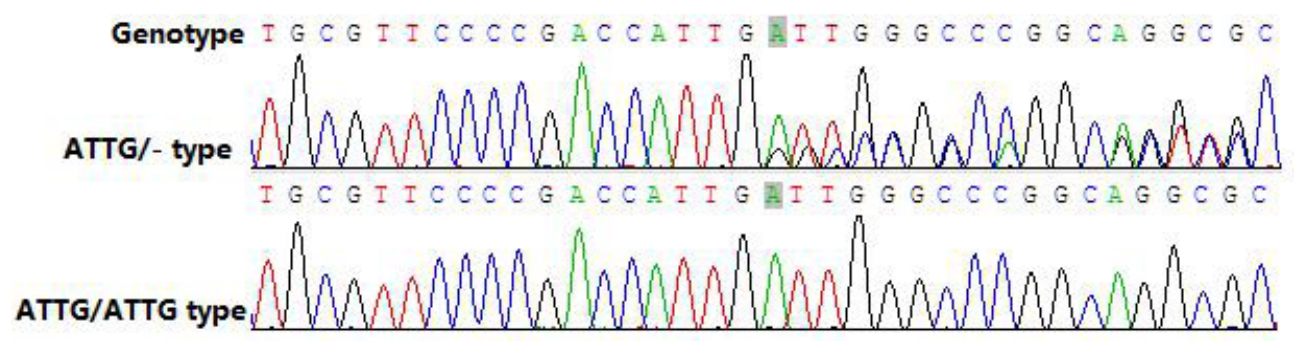

Figure 4. Nuclear factor kappa B (NF- $\kappa B$; rs28720239). ATTG/ATTG type: wild homozygous, ATTG/-: mutant heterozygous (with ATTG deletion). 


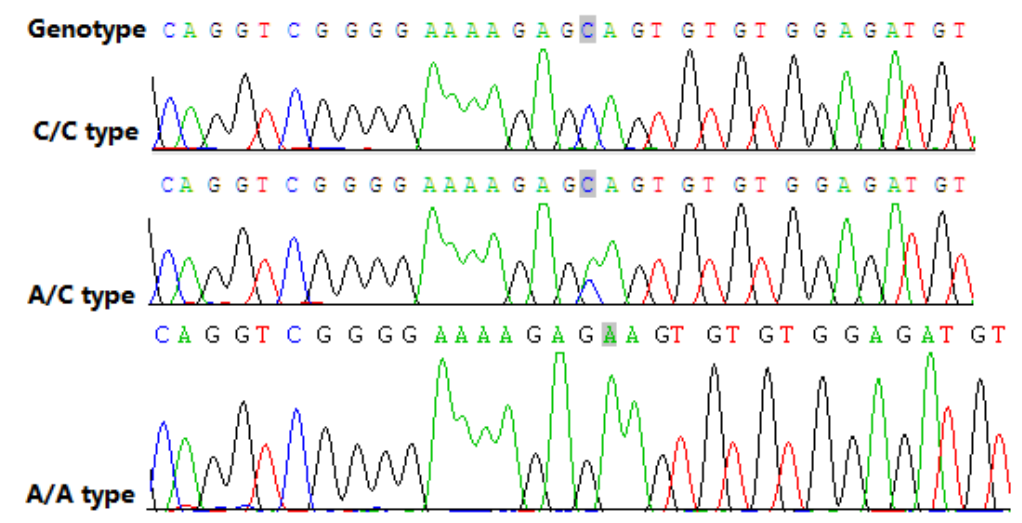

Figure 5. Pulmonary surfactant protein A2 (SP-A2; rs1965708). C/C type: wild homozygous, A/C: mutant heterozygous, A/A: mutant homozygous.

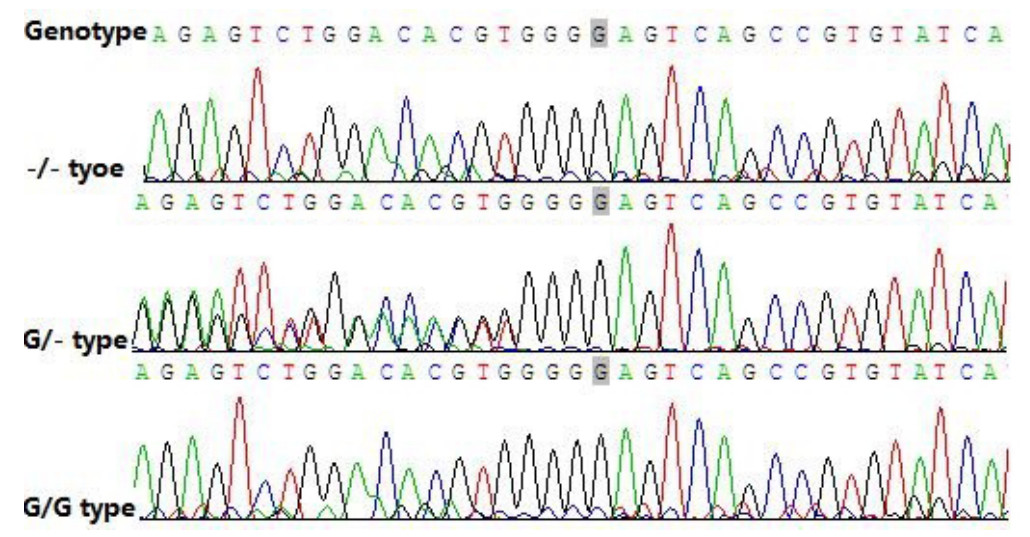

Figure 6. Plasminogen activator inhibitor-1 (PAI-1; rs1799889). -/- type: wild homozygous, G/-: mutant heterozygous, $\mathrm{G} / \mathrm{G}$ : mutant homozygous.

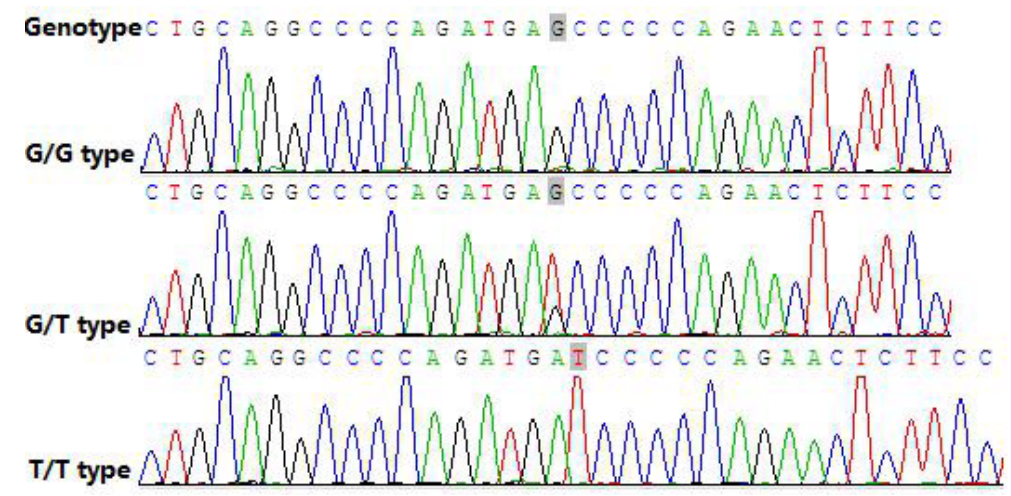

Figure 7. Nitric oxide synthase (NOS; rs199983). G/G type: wild homozygous, G/T: mutant heterozygous, T/T: mutant homozygous. 


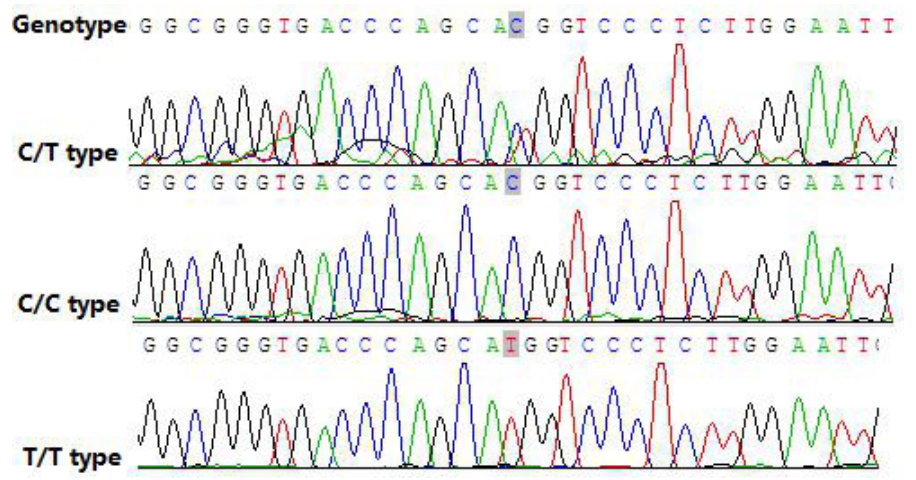

Figure 8. Vascular endothelial growth factor (VEGF; rs3025039). C/C type: wild homozygous, C/T: mutant heterozygous, T/T: mutant homozygous.

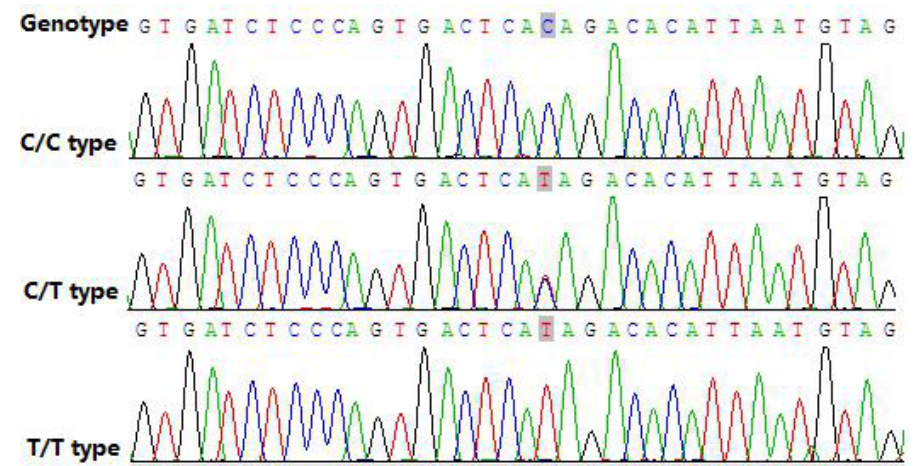

Figure 9. Prolyl hydroxylase (EGLN1; rs480902). C/C type: wild homozygous, C/T: mutant heterozygous, T/T: mutant homozygous.

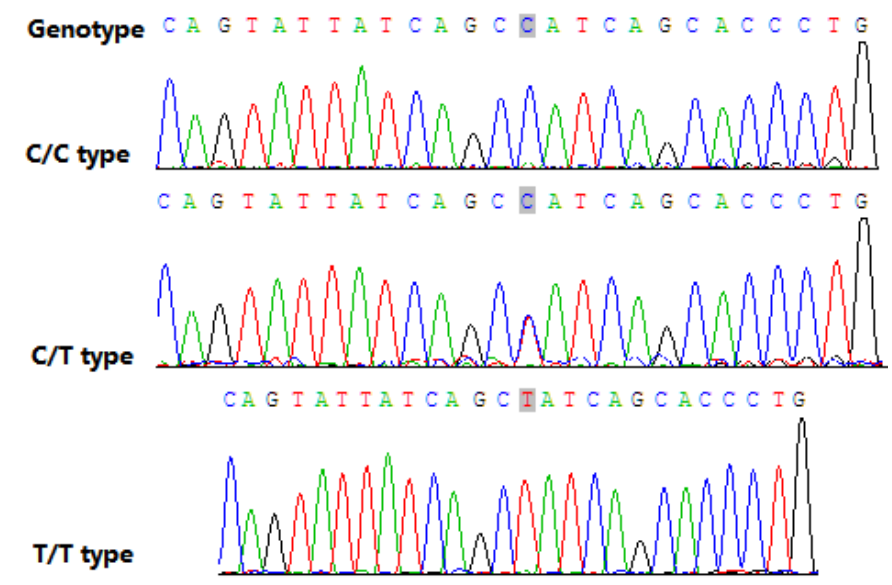

Figure 10. A20 gene promoter region (A20; rs5029924). C/C type: wild homozygous, C/T: mutant heterozygous, T/T: mutant homozygous. 


\section{Analysis of the obtained sequencing results for each site}

The P value of each SNP genotype, calculated by the Hardy-Weinberg balance method, was $>0.05$ in all HAPE subjects (103) and healthy controls (200). This indicated that each group adhered to the Hardy-Weinberg equilibrium principle, and that the samples were representative. The obtained $\mathrm{P}$ values are summarized in Table 1.

Table 1. Results of the Hardy-Weinberg equilibrium tests conducted on SNP loci in hypoxia-related genes.
\begin{tabular}{llcc}
\hline Gene & SNP ID & P value (HAPE group) & P value (Control group) \\
\hline NOS & rs199983 & 0.106 & 0.858 \\
ACE & rs4309 & 0.155 & 0.071 \\
HSP70 & rs1008438 & 0.150 & 0.160 \\
CYP11B2 & rs1799998 & 0.087 & 0.779 \\
SP-A2 & rs1965708 & 0.247 & 0.772 \\
PAI-1 & rs1799889 & 0.601 & 0.076 \\
$V E G F$ & rs3025039 & 0.091 & 0.524 \\
EGLN1 & rs480902 & 0.138 & 0.075 \\
A20 & rs5029924 & 0.060 & 0.268 \\
\hline
\end{tabular}

HAPE, high-altitude pulmonary edema; NOS, nitric oxide synthase; ACE, angiotensin-converting enzyme; HSP70, heat-shock protein 70; CYP11B2, aldosterone synthase 2; SP-A2, surfactant protein A2; PAI-1, plasminogen activator inhibitor-1; VEGF, vascular endothelial growth factor; EGLN1, prolyl hydroxylase; A20, zinc finger protein A20.

No absolute mutant was observed in the rs28720239 locus in patient and control groups. Therefore, this locus could not be applied to the Hardy-Weinberg test, while all other loci adhered to the Hardy-Weinberg principles $(\mathrm{P}>0.05)$.

\section{Genotypes and distribution of alleles between the two groups}

The genotype distribution of each SNP locus, obtained by comparative analysis of the data obtained from 103 HAPE subjects and 200 healthy controls, is summarized in Table 2.

\begin{tabular}{|c|c|c|c|c|c|c|}
\hline \multirow{3}{*}{$\begin{array}{l}\text { SNP ID } \\
\text { rs1799998 } \\
\text { Cases }\end{array}$} & \multicolumn{3}{|c|}{ Genotype (\%) } & \multicolumn{2}{|c|}{ Allele frequency $(\%)$} & \multirow{2}{*}{$\begin{array}{l}\mathrm{P} \text { value } \\
\mathrm{P}=0.065\left(\chi^{2}=5.473\right)\end{array}$} \\
\hline & TT & $\mathrm{CT}$ & $\mathrm{CC}$ & $\mathrm{T}$ & $\mathrm{C}$ & \\
\hline & $12(11.65)$ & $35(33.98)$ & $56(54.37)$ & $59(28.64)$ & $147(71.36)$ & \\
\hline Controls & $9(4.5)$ & $70(35.0)$ & $121(60.5)$ & $88(22.0)$ & $312(78.0)$ & \\
\hline rs4309 & TT & CT & $\mathrm{CC}$ & $\mathrm{T}$ & $\mathrm{C}$ & $\mathrm{P}=0.00\left(\chi^{2}=21.817\right)$ \\
\hline Cases & $9(8.73)$ & $33(32.04)$ & $61(59.22)$ & $51(24.76)$ & $155(75.24)$ & \\
\hline Controls & $4(2.0)$ & $30(15.0)$ & $166(83.0)$ & $28(9.50)$ & $362(90.5)$ & \\
\hline rs1008438 & GG & GT & TT & $\mathrm{G}$ & $\mathrm{T}$ & $\mathrm{P}=0.00\left(\chi^{2}=20.319\right)$ \\
\hline Cases & $4(3.88)$ & $22(21.36)$ & $77(74.76)$ & $30(14.56)$ & $176(85.44)$ & \\
\hline Controls & $1(0.5)$ & $13(6.5)$ & $186(93)$ & $15(3.75)$ & $385(96.25)$ & \\
\hline rs1965708 & AA & $\mathrm{AC}$ & $\mathrm{CC}$ & $\mathrm{A}$ & $\mathrm{C}$ & $\mathrm{P}=0.00\left(\chi^{2}=16.653\right)$ \\
\hline Cases & $2(1.94)$ & $16(15.53)$ & $85(82.52)$ & $20(9.71)$ & $186(90.29)$ & \\
\hline Controls & $0(0.0)$ & $8(4.0)$ & $192(96.0)$ & $8(2.0)$ & $392(98.0)$ & \\
\hline rs1799889 & GG & $\mathrm{G} /-$ & $-/-$ & $\mathrm{G}$ & - & $\mathrm{P}=0.00\left(\chi^{2}=26.677\right)$ \\
\hline Cases & $3(2.91)$ & $25(24.27)$ & $75(72.81)$ & $31(15.05)$ & $175(84.95)$ & \\
\hline Controls & $1(0.5)$ & $11(5.5)$ & $188(94.0)$ & $13(3.25)$ & $387(96.25)$ & \\
\hline rs199983 & TT & GT & GG & $\mathrm{T}$ & $\mathrm{G}$ & $\mathrm{P}=0.037\left(\chi^{2}=6.615\right)$ \\
\hline Cases & $1(0.97)$ & $8(7.77)$ & $94(91.26)$ & $10(4.85)$ & $196(95.15)$ & \\
\hline Controls & $0(0.0)$ & $5(2.5)$ & $195(97.5)$ & $5(1.25)$ & $395(98.75)$ & \\
\hline rs 3025039 & TT & $\mathrm{CT}$ & $\mathrm{CC}$ & $\mathrm{T}$ & C & $\mathrm{P}=0.33\left(\chi^{2}=2.215\right)$ \\
\hline Cases & $2(1.94)$ & $13(12.62)$ & $88(85.43)$ & $17(8.25)$ & $189(91.75)$ & \\
\hline Controls & $1(0.5)$ & $19(9.5)$ & $180(90.0)$ & $21(5.25)$ & $379(94.75)$ & \\
\hline rs480902 & TT & $\mathrm{CT}$ & $\mathrm{CC}$ & $\mathrm{T}$ & $\mathrm{C}$ & $\mathrm{P}=0.001\left(\chi^{2}=14.502\right)$ \\
\hline Cases & $6(5.83)$ & $27(26.21)$ & $70(67.96)$ & $39(18.93)$ & $167(81.07)$ & \\
\hline Controls & $3(1.5)$ & $25(12.5)$ & $172(86.0)$ & $31(7.75)$ & $369(92.25)$ & \\
\hline rs5029924 & TT & CT & $\mathrm{CC}$ & $\mathrm{T}$ & $\mathrm{C}$ & $\mathrm{P}=0.787\left(\chi^{2}=0.478\right)$ \\
\hline Cases & $2(1.94)$ & $12(11.65)$ & $88(85.43)$ & $16(7.77)$ & $190(92.23)$ & \\
\hline Controls & $2(1.0)$ & $24(12.0)$ & $174(87.0)$ & $28(7.0)$ & $372(93.0)$ & \\
\hline
\end{tabular}


As seen in Table 2, significant differences were observed in the SNP loci rs4309, rs1799889, rs1965708, rs1008438, rs480902, and rs199983, between the HAPE and healthy control groups. This difference was not observed for any of the other sites/SNP loci.

\section{DISCUSSION}

HAPE is an idiopathic pneumonedema resulting from hypoxia, or a low-pressure environment, seen at high altitudes. The pathogenesis of HAPE remains to be elucidated. Previous research has revealed that a sharp regional shift to a plateau results in hypoxemia in a majority of the population. In this case, hypoxia inducible factor-1 (HIF-1) is activated, and the vascular endothelial growth factor (VEGF) and inducible nitric oxide synthase (NOS) protein expressions are regulated, by signal transduction systems, such as the active oxygen (R05) system, resulting in increased vascular permeability (Léon-Velarde and Mejía, 2008). Simultaneously, hypoxemia induces water-sodium retention, which in turn increases the volume of the blood, and causes vasodilation of the blood vessels, by regulating the level of expression of the angiotensin-converting enzyme (ACE) and endothelial nitric oxide synthase (NOS), thereby increasing the oxygen supply in the tissues. Hypoxemia, which functions as a stressor, increases the expression of heat-shock proteins, and protects important organs, such as the lungs and the brain, from harm. Previous studies (Sapru et al., 2009; Madách et al., 2010) have reported a correlation between the $4 \mathrm{G} / 5 \mathrm{G}$ polymorphism in the plasminogen activator inhibitor-1 (PAI-1) gene, and the level of expression of pulmonary edema fluid and PAI-1 in the plasma after lung injury. Patients who express the $4 \mathrm{G} / 4 \mathrm{G}$ and $4 \mathrm{G} / 5 \mathrm{G}$ genotype showed a significant increase in PAI-1 expression; such patients suffered illnesses and inflammatory reactions of greater intensity, and demonstrated obvious pulmonary edema. The C1101T, T3192C, and T3234C segments of the pulmonary surfactant protein-A1 (SP-A1), and the segment A3265C of SP-A2, are closely related to HAPE susceptibility (Saxena et al., 2005). The occurrence of HAPE is affected by population and individual differences. A very small portion of the people suffering from HAPE in the same population were observed to migrate to a plateau region (Rupert and Koehle, 2006). Tibetans show greater adaptability to the hypoxic environment of high-altitude regions compared to people belonging to the Han ethnicity; the incidence of HAPE was significantly less in Tibetans compared to that observed in people belonging the Han ethnicity (Cheng et al., 1997; Basu et al., 2007). Genetic factors accounted for approximately $50 \%$ of the factors influencing the adaptation of the human body to highaltitude hypoxic environments (Wu et al., 2005; Wu and Kayser, 2006). In this study, 10 susceptibility genes related to HAPE were identified/screened from the NCBI database; these genes coded for aldosterone synthase 2 (CYP11B2), ACE, heat-shock protein-70 (HSP70), nuclear factor-kappa B $(N F-\kappa B), S P-A 2, P A I-1, N O S, V E G F$, prolyl hydroxylase (EGLN1), and the zinc finger protein A20. Previous studies have shown a significant difference in the expression of HAPE-inducing genes between HAPE patients and people acclimatized to the plateau. The primary reason for this phenotypic difference may be the presence of specific gene-coding proteins closely related to HAPE pathogenesis, in the HAPE-susceptible population (Beall, 2003). Individuals expressing the susceptible genotype can be screened by detecting the genetic markers; such individuals can then avoid exposure to a high-altitude, hypoxic environment, effectively preventing the occurrence of HAPE. This study helped detect the genotypes of 10 SNP loci from 10 genes indicating HAPE susceptibility, using the 
case-control study method, thereby facilitating the search for the HAPE gene-specific marker.

There is a certain degree of insufficiency in the preliminary results obtained in this study. No associations were detected between 4 SNP loci (of the 10 identified loci) and HAPE. Follow-up studies must consider improving the following aspects: the sample quantity must be increased [the prevailing international practice utilizes samples up to an order of magnitude of $10^{3}$ for group correlation analyses (Fang, 2004); increasing the quantity of samples can improve the effectiveness of detection]; the subjects must be homologous; and the number of detected SNP loci must be increased via haplotype analysis. In conclusion, the SNP and disease association study requires a larger sample size; in addition, greater financial and human resources must be invested to facilitate the simultaneous identification and analysis of multiple-SNP sites. In addition, any evidence of an association between SNP loci and disease must be verified by biochemical and gene function analysis, and by a number of other aspects.

HAPE is not an independent disease; its development is controlled by multiple genes as well as environmental factors. Although a number of related genes could be identified by genotyping, the exact molecular mechanisms governing HAPE onset and propagation remains to be elucidated. An in-depth literature survey has suggested the presence of significant genetic heterogeneity in all ethnicities and countries; however, different mutations on a particular gene are known to cause varying effects. Therefore, a susceptibility gene library based on different ethnicities must also be established. This study enables the evaluation of the risk of HAPE in a population expressing different genes, when abruptly exposed to a high-altitude region; based on the results of this study, the related genes could provide information allowing for the identification of susceptible individuals, in order to intervene and prevent the development of HAPE. This enables the identification of HAPE susceptibility in specific groups of people, such as those recruited in the military, climbers, builders, and plateau tourists.

\section{Conflicts of interest}

The authors declare no conflict of interest

\section{ACKNOWLEDGMENTS}

Research supported by the Innovation Scientific Research fund provided by the Cheng-Du Military General Hospital (Project \#2011YG-A23).

\section{REFERENCES}

Basu CK, Banerjee PK, Selvamurthy W, Sarybaev A, et al. (2007). Acclimatization to high altitude in the Tien Shan: a comparative study of Indians and Kyrgyzis. Wilderness Environ. Med. 18: 106-110.

Beall CM (2003). High-altitude adaptations. Lancet 362: s14-15.

Cheng QH, Ge RL, Wang XZ, Chen HX, et al. (1997). Exercise performance of Tibetan and Han adolescents at altitudes of 3,417 and 4,300 m. J. Appl. Physiol. 83: 661-667.

Fang DF (2004). Some noticeable problems in genetic analysis of gene polymorphism and diseases correlation. Natl. Med. J. China 84: 796-798.

Gao YQ (2005). The plateau military medicine. Chongqing Press, Chongqing.

Gao YQ and Gao WX (2009). The manual of plateau health protection. People's Military Medical Press, Peking.

Hotta J, Hanaoka M, Droma Y, Katsuyama Y, et al. (2004). Polymorphisms of renin-angiotensin system genes with highaltitude pulmonary edema in Japanese subjects. Chest 126: 825-830.

Léon-Velarde F and Mejía O (2008). Gene expression in chronic high altitude diseases. High Alt. Med. Biol. 9: 130-139.

Genetics and Molecular Research 14 (3): 11562-11572 (2015)

CFUNPEC-RP www.funpecrp.com.br 
Madách K, Aladzsity I, Szilágyi A, Fust G, et al. (2010). 4G/5G polymorphism of PAI-1 gene is associated with multiple organ dysfunction and septic shock in pneumonia induced severe sepsis: prospective, observational, genetic study. Crit. Care 14: R79.

Manuguerra M, Matullo G, Veglia F, Autrup H, et al. (2006). Multi-factor dimensionality reduction applied to a large prospective investigation on gene-gene and gene-environment interactions. Carcinogenesis 28: 414-422.

Meyer S, Z'graggen BR, Blumenthal S, Borgeat A, et al. (2007). Hypoxia attenuates effector-target cell interaction in the airway and pulmonary vascular compartment. Clin. Exp. Immunol. 150: 358-367.

Patel S and Peacock A (2001). Who will cope at high altitude: is it in the genes? ISMM News 11: 5-8.

Qi Y, Niu W, Zhou W, Hou S, et al. (2008). Correlation between angiotensinogen gene polymorphisms and essential hypertension in Chinese population. J. Hum. Hypertens. 22: 147-150.

Rupert JL and Koehle MS (2006). Evidence for a genetic basis for altitude-related illness. High Alt. Med. Biol. 7: 150-167.

Sapru A, Hansen H, Ajayi T, Brown R, et al. (2009). 4G/5G polymorphism of plasminogen activator inhibitor-1 gene is associated with mortality in intensive care unit patients with severe pneumonia. Anesthesiology 110: 1086-1091.

Saxena S, Kumar R, Madan T, Gupta V, et al. (2005). Association of polymorphisms in pulmonary surfactant protein A1 and A2 genes with high-altitude pulmonary edema. Chest 128: 1611-1619.

The Draft Recommended by the Third National Congress of Chinese Medical Association for Discussion on Plateau Medicine (1996). Plateau disease in China named, typed and diagnosis standard. High Alt. Med. J. 6: 2-4.

Wu T and Kayser B (2006). High altitude adaptation in Tibetans. High Alt. Med. Biol. 7: 193-208.

Wu T, Li S and Ward MP (2005). Tibetans at extreme altitude. Wilderness Environ. Med. 16: 47-54. 